\title{
Direct Observation of the Oxygen Isotope Effect on the In-Plane Magnetic Field Penetration Depth in Optimally Doped $\mathrm{YBa}_{2} \mathrm{Cu}_{3} \mathrm{O}_{7-\delta}$
}

\author{
R. Khasanov, ${ }^{1,2, *}$ D. G. Eshchenko, ${ }^{1,2}$ H. Luetkens, ${ }^{2,3}$ E. Morenzoni, ${ }^{2}$ T. Prokscha, ${ }^{2}$ A. Suter, ${ }^{2}$ N. Garifianov, ${ }^{2}$ M. Mali, ${ }^{1}$ \\ J. Roos, ${ }^{1}$ K. Conder, ${ }^{4}$ and H. Keller ${ }^{1}$ \\ ${ }^{1}$ Physik-Institut der Universität Zürich, Winterthurerstrasse 190, CH-8057, Switzerland \\ ${ }^{2}$ Laboratory for Muon Spin Spectroscopy, PSI, CH-5232 Villigen PSI, Switzerland \\ ${ }^{3}$ Institut für Metallphysik und Nukleare Festkörperphysik, TU Braunschweig, 38106 Braunschweig, Germany \\ ${ }^{4}$ Laboratory for Neutron Scattering, ETH Zürich and PSI, CH-5232 Villigen PSI, Switzerland
}

(Received 20 May 2003; published 6 February 2004)

\begin{abstract}
We report the first direct observation of the oxygen-isotope $\left({ }^{16} \mathrm{O} /{ }^{18} \mathrm{O}\right)$ effect on the in-plane penetration depth $\lambda_{a b}$ in a nearly optimally doped $\mathrm{YBa}_{2} \mathrm{Cu}_{3} \mathrm{O}_{7-\delta}$ film using the novel low-energy muon-spin rotation technique. Spin-polarized low-energy muons are implanted in the film at a known depth $z$ beneath the surface and precess in the local magnetic field $B(z)$. This feature allows us to measure directly the profile $B(z)$ of the magnetic field inside the superconducting film in the Meissner state and to make a straightforward determination of $\lambda_{a b}$. A substantial isotope shift $\Delta \lambda_{a b} / \lambda_{a b}=$ $2.8(1.0) \%$ at $4 \mathrm{~K}$ is observed, implying that the in-plane effective supercarrier mass $m_{a b}^{*}$ is oxygenisotope dependent with $\Delta m_{a b}^{*} / m_{a b}^{*}=5.5(2.0) \%$. These results are in good agreement with magnetization measurements on powder samples.
\end{abstract}

DOI: 10.1103/PhysRevLett.92.057602

PACS numbers: 76.75.+i, 74.72.Bk, 74.78.Bz, 82.20.Tr

One of the fundamental questions concerning the physics of cuprate high-temperature superconductors (HTSC) is whether the electron-phonon interaction plays an essential role in these systems or not. The high values of the superconducting transition temperature $T_{c}$ and the observation of only a tiny oxygen-isotope shift of $T_{c}$ in optimally doped HTSC (see, e.g., [1]) were taken as important arguments to propose alternative pairing mechanisms of purely electronic origin. The conventional phonon-mediated theory of superconductivity is based on the Migdal adiabatic approximation in which the effective supercarrier mass $m^{*}$ is independent of the mass $M$ of the lattice atoms. However, if the interaction between the carriers and the lattice is strong enough, the Migdal adiabatic approximation breaks down and $m^{*}$ depends on $M$ (see, e.g., [2]). The ideal experiment to explore a possible coupling of the supercarriers to the lattice is an isotope effect study of the magnetic field penetration depth $\lambda$. For HTSC, which are superconductors in the clean limit, the in-plane penetration depth $\lambda_{a b}$ obeys the relation

$$
1 / \lambda_{a b}^{2}=\mu_{0} e^{2} n_{s} / m_{a b}^{*}
$$

where $\mu_{0}$ is the permeability of vacuum, $e$ is the elemental charge, $n_{s}$ is the superconducting charge carrier density, and $m_{a b}^{*}$ is the in-plane effective mass of the charge carriers. Since $n_{s}$ was found to be predominantly isotope independent [3-9], isotope effect experiments on $\lambda$ turned out to be a unique tool to investigate unconventional lattice effects in HTSC. Previous oxygen-isotope effect studies of the penetration depth in $\mathrm{YBa}_{2} \mathrm{Cu}_{3} \mathrm{O}_{7-\delta}$ $[3,4], \mathrm{La}_{2-x} \mathrm{Sr}_{x} \mathrm{CuO}_{4}$ [5,6], $\mathrm{Bi}_{1.6} \mathrm{~Pb}_{0.4} \mathrm{Sr}_{2} \mathrm{Ca}_{2} \mathrm{Cu}_{3} \mathrm{O}_{10+\delta}$ [7], and $\mathrm{Y}_{1-x} \operatorname{Pr}_{x} \mathrm{Ba}_{2} \mathrm{Cu}_{3} \mathrm{O}_{7-\delta}$ [4,9] showed, indeed, a pronounced oxygen-isotope dependence of the supercarrier mass. In all these experiments, however, $\lambda$ was determined indirectly from magnetization measurements $[3,4]$, the Meissner fraction [5], magnetic torque [6], and muon-spin rotation ( $\mu \mathrm{SR})$ experiments [9]. In contrast, measurements of the field profile $B(z)$ in the Meissner state allow one to determine $\lambda$ in a straightforward way. Recently, such a direct measurement of $\lambda_{a b}$ in a thin $\mathrm{YBa}_{2} \mathrm{Cu}_{3} \mathrm{O}_{7-\delta}$ film was performed [10] at the Paul Scherrer Institute (PSI, Switzerland) by using the novel low-energy $\mu \mathrm{SR}$ (LE $\mu \mathrm{SR}$ ) technique [11]. In this experiment the muon implantation depth was controlled by the variation of the incoming muon energy, and the magnitude of the field was monitored by the muon-spin precession frequency, as in standard $\mu \mathrm{SR}$.

In this Letter, we report on the oxygen-isotope $\left({ }^{16} \mathrm{O} /{ }^{18} \mathrm{O}\right)$ effect $(\mathrm{OIE})$ on the in-plane magnetic field penetration depth in a high-quality $\mathrm{YBa}_{2} \mathrm{Cu}_{3} \mathrm{O}_{7-\delta}$ film near optimal doping measured directly by LE $\mu \mathrm{SR}$. The experiments revealed that in the Meissner state the magnetic field $B(z)$ in the ${ }^{16} \mathrm{O}$ substituted sample decreases stronger with distance $z$ than in the ${ }^{18} \mathrm{O}$ one, clearly demonstrating that ${ }^{16} \lambda_{a b}<{ }^{18} \lambda_{a b}$.

The samples used for the $\mathrm{LE} \mu \mathrm{SR}$ experiments were high-quality $\mathrm{YBa}_{2} \mathrm{Cu}_{3} \mathrm{O}_{7-\delta}$ films grown in one batch with an area of $2 \times 3 \mathrm{~cm}^{2}$ and a thickness of $600 \mathrm{~nm}$ supplied by Theva [12]. The films were grown by thermal coevaporation of the constituents onto a single-crystal barium titanate substrate with the crystal $c$-axis perpendicular to the plane and the twinned $a b$ planes well aligned with the substrate. The oxygen-isotope exchange was performed by annealing the samples in ${ }^{18} \mathrm{O}_{2}$ gas. In 
TABLE I. Summary of the OIE results for $\mathrm{YBa}_{2} \mathrm{Cu}_{3} \mathrm{O}_{7-\delta}$ extracted from the experimental data (see text for an explanation).

\begin{tabular}{|c|c|c|c|c|c|c|}
\hline Method & Sample & $\begin{array}{l}{ }^{16} T_{c} \\
(\mathrm{~K})\end{array}$ & $\begin{array}{l}{ }^{18} T_{c} \\
(\mathrm{~K})\end{array}$ & $\begin{array}{c}\Delta T_{c} / T_{c} \\
(\%)\end{array}$ & $\begin{array}{c}\Delta \lambda_{a b} / \lambda_{a b} \\
(\%)\end{array}$ & $\begin{array}{c}\Delta m_{a b}^{*} / m_{a b}^{*} \\
(\%)\end{array}$ \\
\hline $\mathrm{LE} \mu \mathrm{SR}^{\mathrm{a}}$ & Thin film & $89.3(1)$ & 89.1(1) & $-0.22(16)$ & $2.8(1.0)$ & $5.5(2.0)$ \\
\hline \multirow[t]{2}{*}{ Magnetization } & Fine powder & $91.66(3)$ & $91.42(3)$ & $-0.26(5)$ & $3.0(1.1)$ & $6.0(2.2)$ \\
\hline & & $91.71(3)^{b}$ & $91.45(1)^{\mathrm{c}}$ & $-0.28(5)$ & $2.4(1.0)$ & $4.8(2.0)$ \\
\hline
\end{tabular}

${ }^{a}$ Because of the low muon rates available at present it was not possible to perform a backexchange experiment.

${ }^{b}$ Results for the back-exchange $\left({ }^{18} \mathrm{O} \rightarrow{ }^{16} \mathrm{O}\right)$ sample.

${ }^{c}$ Results for the back-exchange $\left({ }^{16} \mathrm{O} \rightarrow{ }^{18} \mathrm{O}\right)$ sample.

order to ensure that the ${ }^{16} \mathrm{O}$ and ${ }^{18} \mathrm{O}$ substituted samples are subjects of the same thermal history, the annealing (in ${ }^{16} \mathrm{O}_{2}$ and ${ }^{18} \mathrm{O}_{2}$ ) was performed simultaneously. The exchange process was carried out at $600^{\circ} \mathrm{C}$ during $25 \mathrm{~h}$, followed by slow cooling $\left(20^{\circ} \mathrm{C} / \mathrm{h}\right)$ in order to oxidize them completely. The fraction of the ${ }^{18} \mathrm{O}$ content in the film was estimated to be $95 \%$, corresponding to the ${ }^{18} \mathrm{O}$ content in the initial gas used for the annealing process. The oxygen content $(7-\delta)$ and the quality of the films were estimated from $1 \mathrm{mT}$ field-cooled (FC) SQUID magnetization measurements. The observed $T_{c}$ are ${ }^{16} T_{c}=$ 89.3(1) K and ${ }^{18} T_{c}=89.1(1) \mathrm{K}$. We define $T_{c}$ as the temperature where the linearly extrapolated magnetization slope (in the vicinity of $T_{c}$ ) intersects the zero magnetization line. The transition widths are $\simeq 0.9 \mathrm{~K}$ for both samples. A comparison of the $T_{c}$ 's with the known experimental $T_{c}$ vs $\delta$ curves for ${ }^{16} \mathrm{O}$ and ${ }^{18} \mathrm{O}$ substituted $\mathrm{YBa}_{2} \mathrm{Cu}_{3} \mathrm{O}_{7-\delta}$ [13] yields $\delta=0.150$ (5) for both films. While $\delta$ indicates that the samples are slightly underdoped, the narrow transition shows that the oxygen distribution over the films is quite homogeneous. The results of the OIE on $T_{c}$ are summarized in Table I.

The transverse-field LE $\mu$ SR experiments were performed on the $\pi \mathrm{E} 3$ muon beam line at PSI. A weak external magnetic field of $9.2 \mathrm{mT}$ was applied parallel to the sample surface after the sample was cooled in zero magnetic field from a temperature above $T_{c}$ to $4 \mathrm{~K}$. In this geometry, currents flowing in the $a b$ planes determine the magnetic field profile along the crystal $c$ axis inside the film (see inset of Fig. 1). Spin-polarized muons were implanted at a depth ranging from $20-150 \mathrm{~nm}$ beneath the surface of the film by varying the energy of the incident muons from 3 to $30 \mathrm{keV}$. For each implantation energy a time-differential $\mu$ SR spectrum was measured. The muon implantation depth profile $n(z)$ for the given implantation energy was calculated using the Monte Carlo code TRIM.SP [14]. The reliability of the calculated $n(z)$ was cross-checked by previous LE $\mu$ SR experiments on thin metal layers [15].

The experimental data were analyzed in the following way. For each implantation energy the average value of the magnetic field $\bar{B}$ and correspondent average value of the stopping distance $\bar{z}$ were extracted. The value of $\bar{B}$ was taken from the fit of the time evolution of the muon-spin polarization spectrum by using the Gaussian relaxation function:

$$
a(t)=a_{0} \exp \left(-\sigma^{2} t^{2} / 2\right) \cos (\gamma \bar{B} t+\phi),
$$

where $a_{0}$ is the initial asymmetry, $\sigma$ is the Gaussian relaxation rate, and $\gamma=2 \pi \times 135 \mathrm{MHz} / \mathrm{T}$ is the gyromagnetic ratio of the muon. Note that at our level

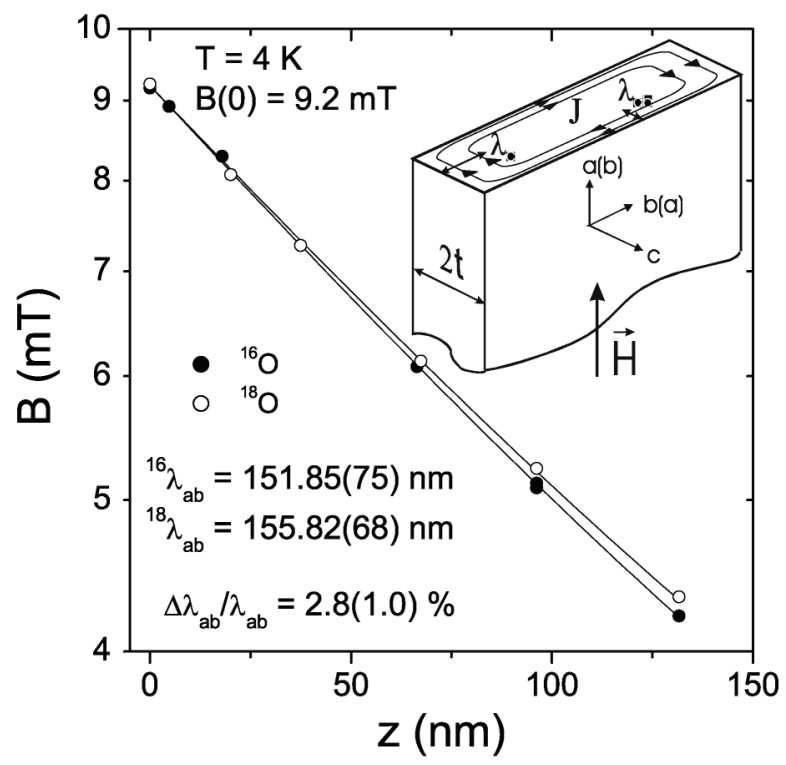

FIG. 1. Magnetic field penetration profiles $B(z)$ on a logarithmic scale for a ${ }^{16} \mathrm{O}$ substituted (closed symbols) and a ${ }^{18} \mathrm{O}$ substituted (open symbols) $\mathrm{YBa}_{2} \mathrm{Cu}_{3} \mathrm{O}_{7-\delta}$ film measured in the Meissner state at $4 \mathrm{~K}$ and an external field of $9.2 \mathrm{mT}$, applied parallel to the surface of the film. The data are shown for implantation energies 3, 6, 10, 16, 22, and $29 \mathrm{keV}$ starting from the surface of the sample. Solid curves are best fits by Eq. (3). Error bars are smaller than the size of symbols. The inset shows schematically the distribution of the screening current in a thin anisotropic superconducting slab of thickness $2 t$ in a magnetic field applied parallel to the flat surface. The screening current $J$ flows preferably parallel to the $a b$ planes, giving rise to an exponential field decay along the crystal $c$ axis. Because of twinning in the $a b$ plane ( $a$ and $b$ axes are not distinguishable) the so-called in-plane magnetic penetration depth $\lambda_{a b}$ is measured. 
of statistics $\left(\sim 5 \times 10^{5}\right.$ events per spectrum) the fit with Eq. (2) to the experimental data satisfies the $\chi^{2}$ criterion ( $\chi^{2}$ less than $n+\sqrt{2 n}, n$ is the number of degrees of freedom minus one, $2 n$ is the variance of the $\chi^{2}$ distribution). The value of $\bar{z}$ was taken as the first moment of the emulated $n(z)$ distribution. Results of this analysis for the ${ }^{16} \mathrm{O}$ and ${ }^{18} \mathrm{O}$ substituted $\mathrm{YBa}_{2} \mathrm{Cu}_{3} \mathrm{O}_{7-\delta}$ films are shown in Fig. 1. The data points for the ${ }^{18} \mathrm{O}$ film are systematically higher than those for the ${ }^{16} \mathrm{O}$ film, showing that ${ }^{16} \lambda_{a b}<{ }^{18} \lambda_{a b}$. The solid lines represent a fit to the $\bar{B}$ data by the function

$$
B(z)=B(0) \frac{\cosh \left[(t-z) / \lambda_{a b}\right]}{\cosh \left(t / \lambda_{a b}\right)} .
$$

This is the form of the classical exponential field decay in the Meissner state $B(z)=B(0) \exp \left(-z / \lambda_{a b}\right)$ [where $B(0)$ is the field at the surface of the superconductor], modified for a film with thickness $2 t$ with flux penetrating from both sides. The value of $z$ was corrected in order to take into account the surface roughness of the films [10], which was taken the same [8.0(5) $\mathrm{nm}$ ] for both samples originating from the same batch. Fits with Eq. (3) to the extracted ${ }^{16} \bar{B}(\bar{z})$ and ${ }^{18} \bar{B}(\bar{z})$ yield ${ }^{16} \lambda_{a b}(4 \mathrm{~K})=$ $151.8(1.1) \mathrm{nm}$ and ${ }^{18} \lambda_{a b}(4 \mathrm{~K})=155.8(1.0) \mathrm{nm}$. Taking into account a ${ }^{18} \mathrm{O}$ content of $95 \%$, the relative shift was found to be $\Delta \lambda_{a b} / \lambda_{a b}=\left({ }^{18} \lambda_{a b}-{ }^{16} \lambda_{a b}\right) /{ }^{16} \lambda_{a b}=$ $2.8(1.0) \%$ at $4 \mathrm{~K}$. Here we mention that $\lambda_{a b}$ obtained from LE $\mu$ SR was determined in a straightforward way. It is extracted from the measured field profile in the Meissner state using only one fit parameter $\lambda_{a b}$.

We also performed additional OIE experiments on $\lambda_{a b}$ based on measurements of the Meissner fraction in fine $\mathrm{YBa}_{2} \mathrm{Cu}_{3} \mathrm{O}_{7-\delta}$ powder. The powder samples were ground for about $60 \mathrm{~min}$ and then passed through a $10 \mu \mathrm{m}$ sieve. The oxygen exchange procedure was analogous to the one described above for the thin film samples. The ${ }^{18} \mathrm{O}$ content in the sample, as determined from the change of the sample weight after the isotope exchange, was found to be $89(2) \%$. The value of the Meissner fraction $f$ was calculated from $1 \mathrm{mT}$ FC SQUID magnetization measurements. The absence of weak links between grains was confirmed by the linear magnetic field dependence of the FC magnetization measured at $5 \mathrm{~K}$ in $0.5,1$, and $1.5 \mathrm{mT}$. The temperature dependence of $\lambda_{\text {eff }}$ (powder average) was calculated from $f$ under the assumption that the sample grains are spherical [16]. The $\lambda_{a b}(T)$ (Fig. 2) was determined from the measured $\lambda_{\text {eff }}(T)$ using the relation $\lambda_{\text {eff }}=1.31 \lambda_{a b}$ which holds for highly anisotropic superconductors $\left(\lambda_{c} / \lambda_{a b}>5\right)$ [17]. Taking into account a ${ }^{18} \mathrm{O}$ content of $89 \%$, the relative shift at $4 \mathrm{~K}$ was found to be $\Delta \lambda_{a b} / \lambda_{a b}=3.0(1.1) \%$ in good agreement with the LE $\mu$ SR data (see Table I). Similar to [6] we also performed a back-exchange experiment. For this purpose the ${ }^{16} \mathrm{O}$ sample was annealed in ${ }^{18} \mathrm{O}_{2}$ gas $\left({ }^{16} \mathrm{O} \rightarrow\right.$ $\left.{ }^{18} \mathrm{O}\right)$ and the ${ }^{18} \mathrm{O}$ sample in ${ }^{16} \mathrm{O}_{2}$ gas $\left({ }^{18} \mathrm{O} \rightarrow{ }^{16} \mathrm{O}\right)$. The

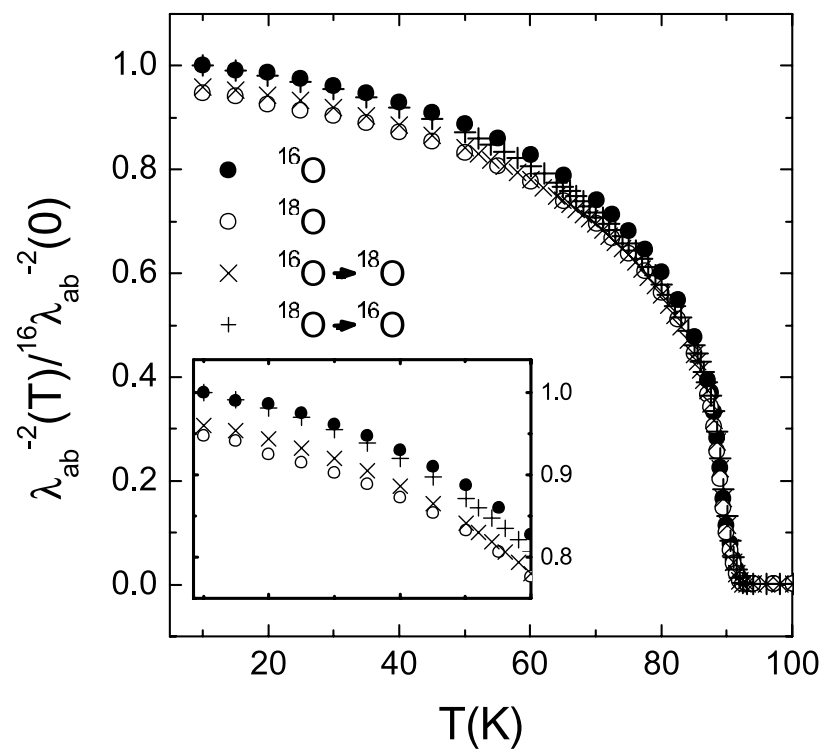

FIG. 2. Temperature dependence of $\lambda_{a b}^{-2}$ normalized by ${ }^{16} \lambda_{a b}^{-2}(0)$ for ${ }^{16} \mathrm{O}$ and ${ }^{18} \mathrm{O}$ substituted $\mathrm{YBa}_{2} \mathrm{Cu}_{3} \mathrm{O}_{7-\delta}$ fine powder samples as obtained from low-field SQUID magnetization measurements. The inset shows the low-temperature region between 10 and $60 \mathrm{~K}$. The reproducibility of the oxygen exchange procedure was checked by the back exchange (crosses).

annealing procedure was analogous to the one described above for the thin film samples. The results of the backexchange experiments are also shown in Fig. 2 (cross symbols). All the results of the OIE on $T_{c}$ and $\lambda_{a b}$ are summarized in Table I. Note that the observed OIE on $\lambda_{a b}$ is remarkable in comparison with the small OIE on $T_{c}$ $\left[\Delta T_{c} / T_{c}=-0.26(5) \%\right]$. This finding is consistent with the empirical "Uemura relation" [18] in a differential way for nearly optimally doped cuprates.

According to Eq. (1), the OIE on $\lambda_{a b}$ is due to a shift in $n_{s}$ and/or $m_{a b}^{*}$ :

$$
\frac{\Delta \lambda_{a b}}{\lambda_{a b}}=\frac{1}{2} \frac{\Delta m_{a b}^{*}}{m_{a b}^{*}}-\frac{1}{2} \frac{\Delta n_{s}}{n_{s}} .
$$

Previous OIE studies in HTSC's [3-9] showed a pronounced OIE on $m_{a b}^{*}$ with negligible OIE on $n_{s}$. In this Letter we provide further evidence of this scenario from measurements of the nuclear quadrupole resonance (NQR) frequency $\left(\nu_{Q}\right)$ of the plane $\left(\nu_{Q}^{p l}\right)$ and the chain $\left(\nu_{Q}^{c h}\right){ }^{63} \mathrm{Cu}$ in ${ }^{16} \mathrm{O}$ and ${ }^{18} \mathrm{O}$ substituted $\mathrm{YBa}_{2} \mathrm{Cu}_{3} \mathrm{O}_{7-\delta}$ powder samples. It is known $[19,20]$ that in HTSC $\nu_{Q}^{p l}$ and $\nu_{Q}^{c h}$ are very sensitive to changes in the density of mobile carriers $n$ in the superconducting $\mathrm{CuO}_{2}$ planes $\left(n^{p l}\right)$ and $\mathrm{CuO}$ chains $\left(n^{c h}\right)$. Both $\nu_{Q}^{p l}$ and $\nu_{Q}^{c h}$ increase with doped hole concentration roughly $20 \mathrm{MHz}$ per one doped hole per $\mathrm{Cu}$ atom [21]. The NQR measurements of ${ }^{63} \mathrm{Cu}$ at $94 \mathrm{~K}$ show that within $5 \mathrm{kHz}$ error bar limits the plane copper and the chain copper NQR frequencies are the same in ${ }^{16} \mathrm{O}$ and ${ }^{18} \mathrm{O}$ substituted $\mathrm{YBa}_{2} \mathrm{Cu}_{3} \mathrm{O}_{7-\delta}$ powder samples (see Table II). This implies that by the 
TABLE II. Results of plane and chain ${ }^{63} \mathrm{Cu} N Q R$ in ${ }^{16} \mathrm{O}$ and ${ }^{18} \mathrm{O}$ substituted $\mathrm{YBa}_{2} \mathrm{Cu}_{3} \mathrm{O}_{7-\delta}$ powder samples at $94 \mathrm{~K}$ (see text for an explanation).

\begin{tabular}{cccccc}
\hline \hline & \multicolumn{2}{c}{${ }^{16} \mathrm{O}$} & \multicolumn{2}{c}{${ }^{18} \mathrm{O}$} \\
& $\begin{array}{c}\nu_{Q} \\
(\mathrm{MHz})\end{array}$ & $\begin{array}{c}\text { Linewidth } \\
(\mathrm{kHz})\end{array}$ & $\begin{array}{c}\nu_{Q} \\
(\mathrm{MHz})\end{array}$ & $\begin{array}{c}\text { Linewidth } \\
(\mathrm{kHz})\end{array}$ & $\begin{array}{c}\Delta \nu_{Q} / \nu_{Q} \\
(\%)\end{array}$ \\
\hline Planes & $31.580(5)$ & $336(10)$ & $31.580(5)$ & $340(10)$ & $0.00(2)$ \\
Chains & $22.050(5)$ & $161(5)$ & $22.050(5)$ & $161(5)$ & $0.00(3)$ \\
\hline \hline
\end{tabular}

oxygen substitution $n^{p l}$ and $n^{\text {ch }}$ change for less than $3 \times$ $10^{-4}$ hole per $\mathrm{Cu}$ atom. In $\mathrm{YBa}_{2} \mathrm{Cu}_{3} \mathrm{O}_{7-\delta}$ one has two plane and one chain $\mathrm{Cu}$ atom in the unit cell. From the NQR measurements we can thus conclude that the change of the hole number per unit cell is less than 3 times $3 \times 10^{-4} \simeq 10^{-3}$ hole. Since in optimally doped $\mathrm{YBa}_{2} \mathrm{Cu}_{3} \mathrm{O}_{7-\delta}$ there is approximately one doped hole per unit cell, the relative change of hole density $\Delta n / n$ at the oxygen exchange must be less than $10^{-3}$. For $\mathrm{YBa}_{2} \mathrm{Cu}_{3} \mathrm{O}_{7-\delta}$ it was shown [22] that $n_{s} \approx n$ at $T<$ $70 \mathrm{~K}$. Based on this observation, we thus can conclude that $\left|1 / 2 \times \Delta n_{s} / n_{s}\right|<0.05 \%$ in Eq. (4). Consequently, the main contribution to the OIE on $\lambda_{a b}$ has to come from the isotope dependence of $m_{a b}^{*}$, so that $\Delta \lambda_{a b} / \lambda_{a b} \simeq$ $\left(\Delta m_{a b}^{*} / \mathrm{m}_{a b}^{*}\right) / 2$. With $\Delta \lambda_{a b} / \lambda_{a b}=2.8(1.0) \%$ we obtain $\Delta m_{a b}^{*} / m_{a b}^{*}=5.5(2.0) \%$ at $4 \mathrm{~K}$ (see Table I).

Note that such an isotope effect on $m_{a b}^{*}$ is not expected for a conventional weak-coupling phonon-mediated BCS superconductor. In fact, in HTSC the charge carriers are strongly coupled to optical phonons, as demonstrated by measurements of the static and high-frequency dielectric constants [23], photoemission [24], and tunneling [25] experiments. Strong interaction between the charge carriers and the lattice ions leads to a breakdown of the adiabatic Migdal approximation [2], and, consequently, the effective supercarrier mass $m^{*}$ depends on the mass of the lattice atoms. To our knowledge there are just a few theoretical models which predict an OIE on $m^{*}$ (see, e.g., Refs. [2,26-28]).

In conclusion, we used LE $\mu$ SR to measure directly the oxygen-isotope $\left({ }^{16} \mathrm{O} /{ }^{18} \mathrm{O}\right)$ effect on the in-plane magnetic field penetration depth $\lambda_{a b}$ in optimally doped $\mathrm{YBa}_{2} \mathrm{Cu}_{3} \mathrm{O}_{7-\delta}$ films. The OIE on $\lambda_{a b}$ at $4 \mathrm{~K}$ was found to be $\Delta \lambda_{a b} / \lambda_{a b}=2.8(1.0) \%$. The intrinsic character of the OIE on $\lambda_{a b}$ was confirmed by back-exchange experiments on fine powders with low-field SQUID magnetization measurements. It is concluded that the OIE arises mainly from the isotope dependence of the in-plane charge carrier mass $m_{a b}^{*}$ with $\Delta m_{a b}^{*} / m_{a b}^{*}=5.5(2.0) \%$ at $4 \mathrm{~K}$. This finding implies that, even in optimally doped cuprate superconductors for which only a small isotope effect on $T_{c}$ is observed, the supercarriers are strongly coupled to the lattice.

This work was partly performed at the Swiss Muon Source $(\mathrm{S} \mu \mathrm{S})$ at the Paul Scherrer Institute (Villigen,
Switzerland). The authors are grateful to A. BussmannHolder, K. A. Müller, T. Schneider, and Z.X. Shen for stimulating discussions. This work was supported by the Swiss National Science Foundation and by the NCCR program Materials with Novel Electronic Properties (MaNEP) sponsored by the Swiss National Science Foundation.

*Electronic address: rustem.khasanov@psi.ch

[1] J. P. Franck, in Physical Properties of High-Temperature Superconductors IV, Singapore, 1994, edited by D. M. Ginsberg (World Scientific, Singapore, 1994), p. 189.

[2] A. S. Alexandrov and N. F. Mott, Int. J. Mod. Phys. B 8, 2075 (1994).

[3] G. M. Zhao and D. E. Morris, Phys. Rev. B 51, 16487 (1995).

[4] G. M. Zhao, J.W. Ager, and D. E. Morris, Phys. Rev. B 54, 14982 (1996).

[5] G. M. Zhao et al., Nature (London) 385, 236 (1997).

[6] J. Hofer et al., Phys. Rev. Lett. 84, 4192 (2000).

[7] G. M. Zhao et al., Phys. Rev. B 63, 220506 (2001).

[8] G. M. Zhao, H. Keller, and K.Conder, J. Phys. Condens. Matter 13, R569 (2001).

[9] R. Khasanov et al., J. Phys. Condens. Matter 15, L17 (2003).

[10] T. J. Jackson et al., Phys. Rev. Lett. 84, 4958 (2000).

[11] E. Morenzoni et al., Phys. Rev. Lett. 72, 2793 (1994).

[12] www.theva.com, info@theva.com

[13] D. Zech, Ph.D. thesis, University of Zurich, 1995.

[14] W. Eckstein, Computer Simulations of Ion-Solid Interactions (Springer-Verlag, Berlin, 1992).

[15] E. Morenzoni et al., Nucl. Instrum. Methods Phys. Res., Sect. B 192, 254 (2002).

[16] D. Shoenberg, Proc. R. Soc. London, Ser. A 175, 49 (1940).

[17] V. I. Fesenko, V. N. Gorbunov, and V. P. Smilga, Physica (Amsterdam) 176C, 551 (1991).

[18] Y. Uemura et al., Phys. Rev. Lett. 62, 2317 (1989); Y. Uemura et al., ibid. 66, 2665 (1991).

[19] T. Imai et al., Phys. Rev. Lett. 70, 1002 (1993).

[20] J. Karpinski et al., Phys. Rev. B 64, 094518 (2001).

[21] R. Stern et al., Phys. Rev. B 50, 426 (1994).

[22] A. T. Fiory et al., Phys. Rev. Lett. 65, 3441 (1990).

[23] A. S. Alexandrov and A. M. Bratkovsky, Phys. Rev. Lett. 84, 2043 (2000).

[24] Z.X. Shen et al., cond-mat/0108381.

[25] Yu. G. Ponomarev et al., Solid State Commun. 111, 513 (1999).

[26] D. J. Scalapino, R.T. Scalettar, and N. E. Bickers, in Proceedings of the International Conference on Novel Mechanisms of Superconductivity, New York, 1987, edited by S. E. Wolf and V. Z. Kresin (Plenum, New York, 1987), p. 475.

[27] C. Grimaldi, E. Cappelluti, and L. Pietronero, Europhys. Lett. 42, 667 (1998).

[28] A. Bussmann-Holder and R. Micnas, J. Supercond. 15, 321 (2002). 